\title{
Cell Culture Microfluidic Biochips: Experimental Throughput Maximization
}

\author{
Wajid Hassan Minhass, Paul Pop, Jan Madsen \\ Dept. of Informatics and Mathematical Modeling \\ Technical University of Denmark \\ DK-2800, Kgs. Lyngby, Denmark
}

\author{
Mette Hemmingsen, Peder Skafte-Pedersen, Martin Dufva \\ Dept. of Micro- and Nanotechnology \\ Technical University of Denmark \\ DK-2800, Kgs. Lyngby, Denmark
}

\begin{abstract}
Microfluidic biochips offer a promising alternative to a conventional biochemical laboratory, integrating all necessary functionalities on-chip in order to perform biochemical applications. Researchers have started to propose computer-aided design tools for the synthesis of such biochips. Our focus in this paper is on the optimization of how a biochemical application is performed on a biochip. In this paper, we consider cell culture biochips, where several cell colonies are exposed to soluble compounds and monitored in real-time to determine the right combination of factors that leads to the desired results. These biochips have high research potential, e.g., cancer research, stem cell, drug discovery. The application considered is a full-factorial experimental design, where all possible combinations of compounds are applied. We are interested to automatically synthesize (currently done manually) the settings of an experimental design, consisting of decision on the placement pattern of cell colonies and the insertion schedule of compounds such that the biochip throughput is maximized, thus increasing the system productivity, saving time and reducing costs. We have proposed a Simulated Annealing metaheuristic for experimental design generation for the cell culture microfluidic biochips, and we have evaluated our approach using multiple experimental setups.
\end{abstract}

Keywords: Biochips, Throughput optimization, Cell culture

\section{INTRODUCTION}

During the last decade, microfluidic biochips have become an actively researched area. By miniaturizing the macroscopic chemical and biological processes to a sub-millimeter scale, microfluidic systems enable the integration of various assays onto a single chip. The miniaturization also results in reduced required reagent volumes, saving material costs, and higher-precision analyses compared to the conventional methods. There are two technologies for the microfluidic biochips: flow-based [7] and droplet-based [9]. In flow-based microfluidic biochips, the liquid flows continuously through pre-defined micro-channels, with the flow being controlled through valves and pumps [7].

In this paper we are interested in flow-based cell culture biochips. Cell culturing provides biological insights into cells and tissues showing great promise for biomedical and pharmaceutical research. Robotics-based automated cell culture systems are being used today to increase the throughput by using their ability to manage a large number of experiments, e.g., a cell culture system featuring a CRS 465 robotic arm can handle up to 504 micro-plates [15]. Flow-based biochips offer a promising alternative to robotic systems [11]. Biochips can mimic both the complex biochemistries and the geometry of environments found in organisms. At the same time, transport of fluids and soluble factors is regulated through the microfluidic channels creating new opportunities for the spatial and temporal control of cell growth and stimuli [1].
We assume that the chips have been manufactured, possibly using the state-of-the-art computer-aided design (CAD) tools. For example, incipient research has proposed hierarchical modeling and simulation framework for flow-based biochips, from the component-level to the system-level [8]. CAD support for the physical-level has also been proposed [10]. Several microfluidic cell culture platforms have been proposed. In this paper we will consider the biochip architecture presented in Section II.A, which is currently being developed as an extension of the biochip presented in [4]. It offers more fluidic inputs compared to the other proposed architectures [13][14] and real-time fluorescence microscopy observation. The system is programmable, providing simultaneous software control of the pumps and the microscope for automated image analysis. Our focus in this paper is on the optimization of how the biochemical application is performed on these biochips.

Based on the application objectives, different types of experimental designs (i.e., how to setup and run the experiment) can be chosen. In this paper, we are interested in full factorial experimental design, where data is obtained for all possible combinations of factors ${ }^{1}$ (i.e., influencing agents that can be varied by the engineer). This allows the impact of each factor and interaction of the factors to be analyzed.

Biomedical research can be divided into two methods: (1) millions of compounds are screened for effects on one target (e.g., a cell or an enzyme). This is the first step in drug discovery. Since there are millions of compounds to screen for, only a fractional factorial design is utilized. Once the lead compounds have been identified, a full factorial design is initiated. Full factorial experiments are expensive in terms of time and cost and hence need to be performed using high throughput techniques to conserve resources [12]. (2) The other method is to delineate more complex relationships in biological systems. In this case the question is "which factors affect which targets", e.g., which cytokines have effects on which immune cells, which transcription factors bind to and activate which genes. The number of combinations that need to be tested could be huge in some situations and this requires high throughput techniques.

The cell culture biochip architecture, shown in Fig. 1, provides the opportunity for performing these high throughput experiments. An experiment is defined as the exposure of a cell colony to a sequence of compounds and monitoring its response. The biochip shown in the figure can hold 64 cell colonies and thus 64 experiments simultaneously $(8 \times 8 \mathrm{ma}-$ trix, see Section II.A for details). All the experiments being

The term factors and compounds will be used interchangeably in this paper 
carried out in a chip are collectively considered as one experimental stage. An experiment is considered unique if it is not repeated anywhere on the chip. The biochip throughput depends on the number of unique experiments carried out on the chip. Note that several experimental stages might be necessary to achieve a certain target objective. A new chip (or the same chip might be washed and reused) has to be used for the next experimental stage.

An experimental stage has two steps: (1) in the first step the cell colonies are inserted in the chip. The placement is fixed for an entire experimental stage. (2) In the second step, the soluble factors are inserted using either the top-to-bottom route (insertion from the top inlets: $B_{1}$ to $B_{8}$ in Fig. $1 \mathrm{~b}$, exit using the bottom outlets) or the left-to-right route. When a factor is inserted from an inlet (e.g., $B_{2}$ ), it traverses through all colonies in its way till it reaches the outlet (e.g., the colony set $\left(C^{*}, C_{9}, C_{7}, C_{1}, C_{6}, C_{1}, C_{6}, C^{+}\right)$in the second column), influencing all the 8 experiments (every cell colony is a separate experiment). The soluble factors are manually placed in inlet reservoirs. However, they are transported to the biochip automatically using pumps according to the insertion schedule determined offline, i.e., the schedule is decided before the experiment is performed. The pumps are controlled using a programmable custom-made electrical controller. The cell placement pattern (1) and the factor insertion schedule (2) determine the number of unique experiments in an experimental stage. The serialized insertion, especially, reduces the probability of having a high number of unique experiments.

Today the experimental design, i.e., deciding the placement pattern of the cell colonies on the biochip and the schedule of the stimuli insertion (which compounds to insert, in what sequence, and from which inlets), is done manually resulting in undesired repetitions of experiments on the chip, reducing the overall throughput. We propose an optimization approach to automate the cell culture biochip experimental design such that the experimental throughput is maximized. Maximizing the throughput increases system productivity, saving time (one cell culture experiment can take days to complete) and reducing costs, the purified proteins and compounds used in the experiments are highly expensive.

\section{SYSTEM MODEL}

\section{A. Biochip Architecture}

The prototype platform for the programmable microfluidic system used for cell culturing and real-time monitoring [4], shown in Fig. 1a, is equipped with three miniaturized 8-channel peristaltic pumps together with the inlet and outlet reservoirs. The pumps are controlled through a custom-made electrical controller. The chip holder allows exchangeable chips to be readily snapped on to the fluidic interconnections.

Fig. 1b shows the biochip architecture model. A biochip architecture $\mathcal{M}$ is thus represented by an $N \times M$ matrix ( $N$ rows representing $N$ chambers, where each chamber hosts $M$ cell colonies). Each element of the $N \times M$ matrix hosts a cell colony.

Before starting an experiment, the locations on the chamber where the cell colonies are to be placed are tagged using DNA spotting technique. Then, to carry out the experiment, first, valve $V_{2}$ is opened and valve $V_{1}$ is closed, allowing

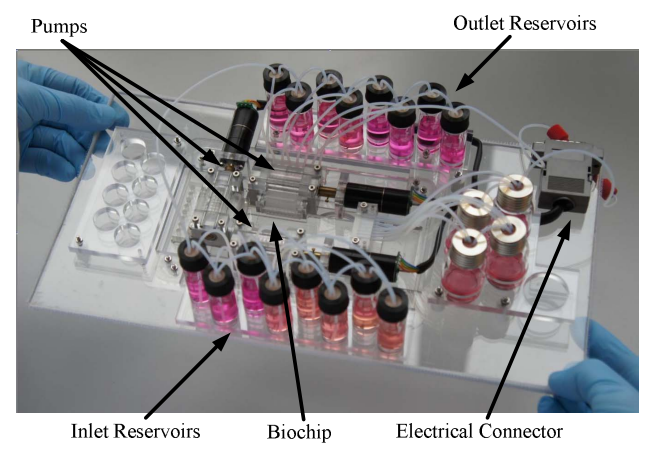

(a) Prototype platform [4]

(b) Biochip architecture model

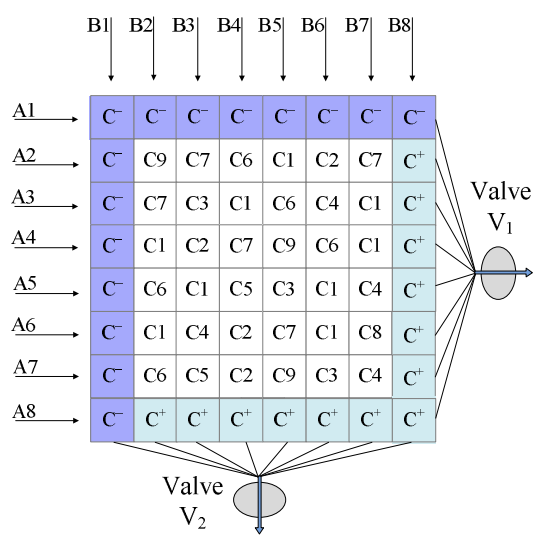

Fig. 1: Biochip Architecture

different cell types to be pumped into the large chamber. The biochip uses the laminar flow property, a distinguished category in the liquid flow classification [3], defined as the flow of fluids in parallel layers without any disruption between the layers. Thus, eight corresponding stripes of cells are produced. After sedimentation, cells adhere to the bottom of the chip according to the DNA spotting. Then, valve $V_{2}$ is closed and valve $V_{1}$ is opened, allowing eight soluble compounds to perfuse over the cells in the perpendicular direction creating simultaneous experiments in the chip. It is also possible to insert soluble compounds using the same inlets from which the cells were initially inserted (valve $V_{1}$ closed, $V_{2}$ open), but only one type of inlets can be open at a time.

Positive $\left(C^{+}\right)$and negative $(C)$ controls, provided by specific cell colonies depending on the application, are used to ensure the quality of compounds being inserted into the chamber and to guarantee that the conditions in the chamber are uniform [5]. The actual size of the chip here is $8 \times 8$, but since the controls need to be placed at the boundary locations so the active area is reduced to $6 \times 6$.

\section{B. Experimental Design}

Exposure of a cell colony to a sequence of soluble compounds and monitoring its reaction is termed as an experiment. All the experiments being carried out in a chip are collectively considered as one experimental stage. Fig. $2 a-2 c$ shows a $6 \times 6$ chip with an active area of $4 \times 4$. The chip is used to carry out experiments in $n=3$ experimental stages, (a) to (c).We consider a set $C$ of two cell colonies $\left(C_{1}\right.$ and $C_{2}$ ) and a set $X$ of three compounds (factors) to be inserted $\left(F_{1}\right.$ to $\left.F_{3}\right)$. We would like to expose the cells to the 


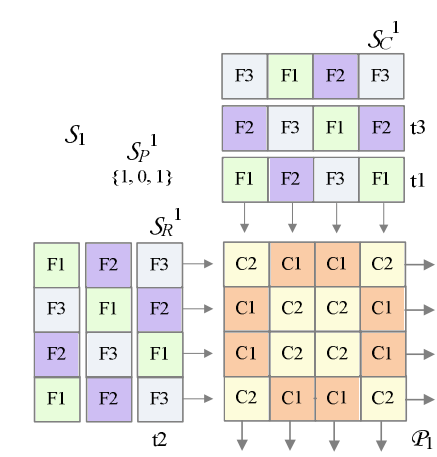

Straightforward solution: (a) $\Omega_{1}$

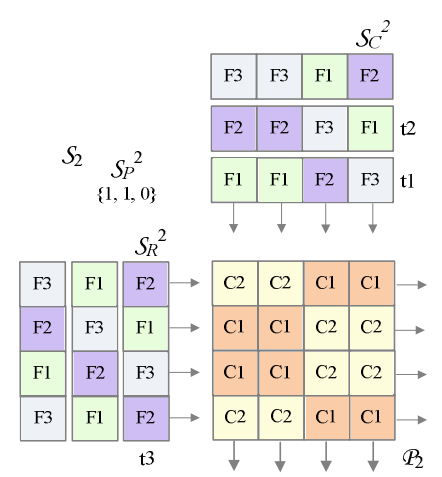

(b) $\Omega_{2}$

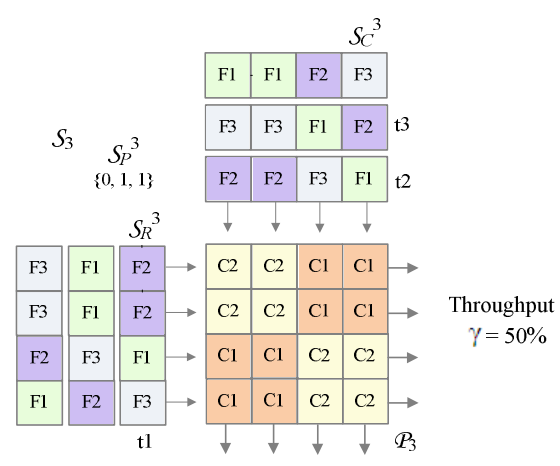

(c) $\Omega_{3}$

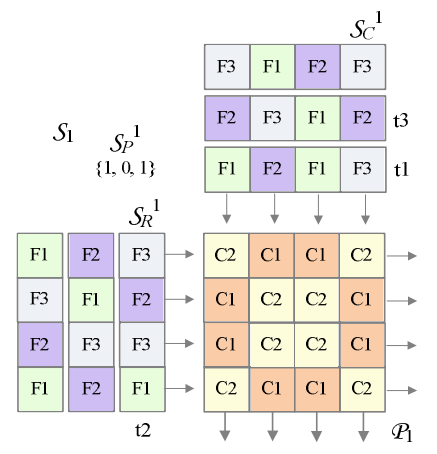

Optimized solution

(d) $\Omega_{1}$

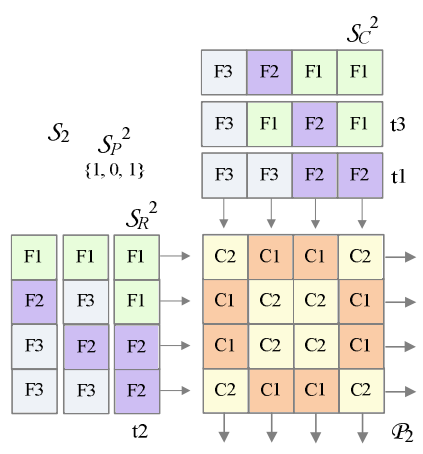

(e) $\Omega_{2}$

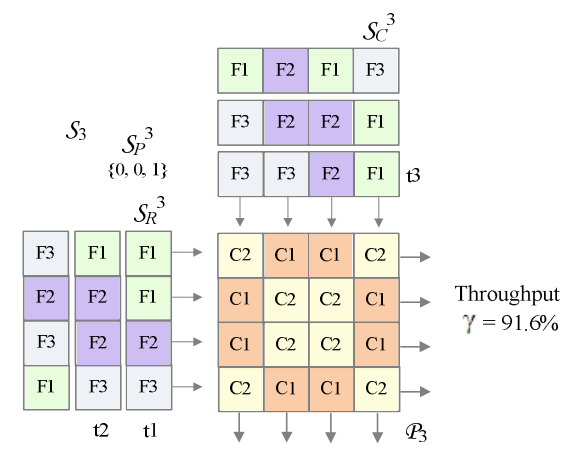

(f) $\Omega_{3}$
Fig. 2: Motivational Example

compounds, in any order. The exposure time per compound is fixed. However, since we allow the compound to be repeated in an exposure sequence, we can thus increase the exposure time for the same compound. The specifications are typically based on the type and nature of the experiment being performed and the practical limitations if any, e.g., maximum number of experimental stages may be decided based on the available budget (experiments are highly expensive).

The placement of cell colonies on the biochip is given by the matrix $\mathcal{P}$, see for example $\mathscr{P}_{1}$ in Fig. 2 a. The sequence of compounds is captured by the schedule $S$, composed of $<S_{R}, S_{C}, S_{P}>$. The compounds can be inserted either from left to right (row-wise) or from top to bottom (column-wise). Two matrices $S_{R}$ and $S_{C}$ represent the compound placement at the insertion point, row-wise and column-wise, respectively. Compounds are placed in the inlet reservoirs, see Fig la. Using pumps and valves, they can be brought at any of the inputs into the chamber, column-wise $\left(B_{1}-B_{8}\right)$ or row-wise $\left(A_{1}-A_{8}\right)$, see Fig. $1 \mathrm{~b}$. The set $S_{P}$ represents the sequence in which the compounds are inserted. A " 1 " in $S_{P}$ marks a column-wise insertion whereas a " 0 " represents a row-wise insertion, see for example $S_{1}=<S_{R}{ }^{1}, S_{C}{ }^{1}, S_{P}{ }^{1}>$ in Fig. 2 a.

Through experiments, we have to find out the right sequence of compounds that provide the desired result for a specific cell colony. To maximize the number of combinations applied to a cell colony, it is important to perform as many unique experiments on the chip as possible. A unique experiment is one which is not repeated (in another part of the chip, or during another experimental stage). Using the placement $\mathcal{P}_{1}$, the schedule $S_{1}=\left\langle S_{R}{ }^{1}, S_{C}{ }^{1}, S_{P}{ }^{1}>\right.$, depicted in Fig. 2a, will result in the following exposure sequence for the cell colony $C_{2}$ placed in the top-left corner: $F_{1}$ (from $S_{C}$ ),
$F_{3}$ (from $S_{R}$ ), $F_{2}$ (from $S_{C}$ ), denoted as " 132 ". This will result in the following experiments (sequence of the experiments listed below is a row by row listing from the matrix):

$C_{1}: 233,331,122, \underline{122}, 112, \underline{112}, \underline{233}, \underline{331}$

$C_{2}: 132,132,223,321,213,311,132,132$

where we have underlined the experiments that have been repeated. Repetition of experiments results in inefficient utilization of the chip. This results in diminished system productivity and enhanced expenses, both in terms of time and cost. Therefore, efficient experimental design is essential. Note that although our model uses two matrices, $S_{R}$ and $S_{C}$ to capture the compounds, only one such matrix is used in the implementation. The set $S_{P}$ will determine if a particular row in this matrix is fed row-wise or column-wise.

Considering an $N \times M$ biochip $\mathcal{M}$, the maximum number of unique experiments that can be carried out in one experimental stage is given by the active chip area: $N^{\prime} \times M^{\prime}$ where $N^{\prime}=(N-2)$ and $M^{\prime}=(M-2)$. Thus, for Fig. 2, the maximum utilization $U_{\max }$ of the biochip is: $U_{\text {max }}=4 \times 4=16$. The maximum number of combinations possible for a given colony depends on the experimental specifications, captured by the set of compounds $X$ and the number of compounds $I$ in an exposure sequence and is equal to $(|X|)^{I}$, where $|X|$ is the number of elements in the set $X$. For the experiments in Fig. $2 \mathrm{a},(|X|)^{I}=27$, the total number of combinations of $I=3$ items taken from a set of three elements $X=\left\{F_{1}, F_{2}, F_{3}\right\}$. The number of possible compound combinations $(|X|)^{I}$ for a certain cell colony is typically larger than the maximum chip capacity $U_{\max }$, which has to be divided among several cell colonies. For the placement in Fig. 2a, colony $C_{1}$ can use only half of the total capacity, $U_{\max } / 2=8$. Thus, to achieve a wider coverage from the set of 27 unique experiments per colony, it is imperative to have more experimental stages even if the chip is fully utilized for the first stage, i.e., all 16 experiments ( 8 per colony) carried out in 
the first stage are unique. In the current case, the biochemist has specified 3 experimental stages. This means that in the ideal case, 24 out of the 27 unique experiments can be achieved for each colony.

The same biochip may be washed and manually reloaded with cell colonies for reuse in multiple experimental stages.

\section{PROBLEM FORMULATION}

The problem we are addressing in this paper can be formulated as follows. Given (1) a cell culture biochip architecture model $\mathcal{M}$ consisting of an $N \times M$ matrix representing the cell culture chip chamber, (2) a set of compounds $X$ that are to be inserted from the inlets, (3) a set of cell colonies $C$ that are to be placed on the cell culture chamber, (4) the number of compounds per exposure sequence $I$, and (5) the total number of experimental stages $n$ to be performed, we are interested in designing each experimental stage $\Omega_{i}, i=1 \ldots n$, such that the experimental throughput $\gamma$ is maximized.

Designing an experimental stage $\Omega_{i}<\mathscr{P}_{i}, S_{i}>$ means deciding for each stage $i$ on: (1) the placement $\mathscr{P}_{i}$ of the cell colonies from the set $C$ and (2) the compound exposure schedule $S_{i}=<S_{R}{ }^{i}, S_{C}{ }^{i}, S_{P}{ }^{i}>$. The throughput is defined as:

$$
\gamma=\frac{|E|}{\min \left(n \times U_{\max },(|X|)^{I \times|C|)}\right.}
$$

where $|E|$ is the number of elements in the set $E$ of unique experiments over $n$ stages, considering the synthesized design $\Omega$. The maximum number of unique experiments is bounded by (i) the chip capacity, $n \times U_{\max }$, and by (ii) the total number of possible combinations, considering all cell colonies $C,(|X|)^{I} \times|C|$, whichever of the two values is smaller. Thus, $\gamma$ represents a percentage of unique experiments obtained through our design $\Omega$ to the maximum possible, given the chip capacity or the number of combinations. Note that a $100 \%$ throughput might not be possible to obtain, and we have no way of knowing maximum possible throughput unless we obtain the optimal design.

Let us use the example in Fig. 2 to illustrate our problem. In Fig. 2, we have $N^{\prime}=M^{\prime}=6-2=4, C=\left\{C_{1}, C_{2}\right\}, X=\left\{F_{1}\right.$, $\left.F_{2}, F_{3}\right\}, I=3$ and $n=3$. Let us suppose we start from the configuration shown in Fig. $2 \mathrm{a}$, where we assume that the placement $\mathscr{P}_{1}$ and schedule $S_{1}$ have been decided as depicted in the figure. All the experiments obtained in this first stage $\Omega_{1}=\left\langle\mathscr{P}_{1}, S_{1}\right\rangle$ have been listed in eq. (1).

As shown, for $C_{2} 5$ out of the total 8 experiments carried out are unique and for $C_{1}$ only 4 experiments are unique. The remaining experiments (underlined) are repetitions. Thus, the chip capacity for this stage is poorly utilized.

In order to increase to number of unique experiments we have to carefully design the second stage $\Omega_{2}=\left\langle\mathscr{P}_{2}, S_{2}\right\rangle$. For the second stage, the same biochip can be used but, as stated earlier, it needs to be washed and reloaded with cell colonies at the decided placements. A simple way to generate a stage $\Omega_{i}$ is to modify a previous stage $\Omega_{i-1}$ using a certain set of rules. We have decided to roll over the placement $\mathscr{P}_{i-1}$ and the contents of the schedule $S_{i-1}$ in the hope to increase the number of unique combinations. Thus, we obtain the stage design $\Omega_{2}$ from Fig. 2b, where $\mathscr{P}_{1}, S_{R}{ }^{1}, S_{C}{ }^{1}$ and $S_{P}{ }^{1}$ are all rolled to the right, i.e., all the contents are shifted right and the ones in the right most position are moved to the first entries on the left. The experiment is run again and the following experiments are generated:

$C_{1}: 232,312,121, \underline{121}, 123, \underline{123}, \frac{232}{311}, \frac{312}{122}$

The experiments underlined are repeated in the same stage and the ones marked in bold have already been covered in the previously conducted experimental stage listed in eq. (1). Each of $C_{1}$ and $C_{2}$ now has 4 more unique experiments, whereas ideally, 8 unique experiments could have been achieved in this experimental stage. Again, the chip capacity has been utilized poorly for this stage as well.

More variations are now required in the experimental settings in order to increase the probability of covering more unique experiments. Thus, for the third experimental stage, a top-to-bottom roll is performed on the settings in Fig. 2b, i.e., all the contents are shifted one step towards the bottom and the ones at the bottom position are moved to the first entries on the top. Since the top-to-bottom roll cannot be performed on the $S_{P}^{2}(1-\mathrm{D}$ array), it is rolled to the right instead. The new settings are shown in Fig. 2c. The generated experiments are:

$C_{1}: 231,212, \underline{231}, \underline{212}, \mathbf{1 2 3}, \mathbf{1 2 3}, 323, \underline{323}$

$C_{2}$ : 223, 223, 223, 223, 131, 112, 331, 312

As shown above, only 3 new unique experiments for $C_{1}$ and 4 for $C_{2}$ have been generated.

For all three experimental stages listed above, the joint experimental throughput $\gamma$ can be calculated using eq. 2 as: $(4+5+4+4+3+4) / 48=50 \%$, Here the denominator 48 is calculated from $\min \left(3 \times 16,(3)^{3} \times 2\right)$. Thus, collectively, only $50 \%$ of the system experimental capacity is being utilized.

A lower chip throughput translates in reduced system productivity and wastage of resources. We are interested in designing an optimized experimental design $\Omega$ such that the throughput $\gamma$ is maximized. Such an optimized $\Omega$ is presented in Fig. $2 \mathrm{~d}-2 \mathrm{f}$, where the throughput obtained is $91.6 \%$ instead of $50 \%$. Fig. $2 \mathrm{~d}-2 \mathrm{f}$ show the settings for the three experimental stages designed for higher throughput. The experiments generated using these settings are:

$C_{1}: 233,131,122,322,132,332,213,111$

$C_{2}: 132,332,223,121,233,131,112,312$

$C_{1}: 311,212,313,211,323,221,321,222$

$C_{2}: 313,211,311,212,321,222,323,221$

$C_{1}: 113,112,123,121,223, \mathbf{2 2 1}, 333, \mathbf{3 3 2}$

$C_{2}: 113,111,123,122, \mathbf{2 2 3}, \mathbf{2 2 2}, 333,331$

Stage 1

Stage 2

resulting in $\gamma=(8+8+8+8+6+6) / 48=91.6 \%$.

Stage 3

Finding such a solution, even for this simple case, can be quite tedious and complex. The next section presents our optimization strategy for automatically deriving the experimental design $\Omega$ which maximizes the throughput $\gamma$.

\section{EXPERIMENTAL THROUGHPUT OPTIMIZATION}

The problem presented in the previous section is NP-complete. To maximize the throughput $\gamma$ over all the $n$ stages, we would have to simultaneously optimize all the matrices $\Omega_{i}=\left\langle\mathscr{P}_{i}, S_{i}\right\rangle$ for all stages $i=1 \ldots n$, since the uniqueness of an experiment is defined across all stages.

Instead, our Experimental Throughput Optimization 
(ETO) strategy, presented in Fig. 3, is to optimize the stages incrementally, one stage at a time. Thus, when optimizing a stage $\Omega_{i}$, we aim to maximize the number of unique experiments considering all the experiments generated in stages $\Omega_{1}$ to $\Omega_{i}$. This approach does not guarantee to find the optimal experimental design, but as the evaluation in Section $\mathrm{V}$ shows, it can obtain very good results in a short time.

The initial solution is generated using a heuristic presented in Section IV.A (line 1 in Fig. 3). The final stages are generated iteratively (lines 3-6 in Fig. 3) using an approach based on the Simulated Annealing (SA) meta-heuristic (presented in detail in Section IV.B). SA takes as input the configuration of the previous stage $<\mathscr{P}_{i-1}, S_{i-1}>$ and the set of unique experiments $E$ generated so far, and determines the configuration $\Omega_{i}=\left\langle\mathscr{P}_{i}, S_{i}\right\rangle$ such that the number of unique experiments generated are maximized. The set $E$ contains all the unique experiments generated, from $\Omega_{1}$ to $\Omega_{i-1}$.

\section{A. Initial Solution}

The initial configuration is created using a Descend-Ascend scheme. The configuration starts by placing the colony/ compound with the highest value identifier (e.g., $C_{5}$ if we have 5 colonies) at the top left location. Then, the second highest identifier $\left(C_{4}\right)$ (descend) is placed at the next row position and so on. At the end of each row, the sequence moves to the start of the next row. On reaching the smallest value identifier (e.g., $C_{1}$ ), the scheme switches to ascend, i.e., the identifier placement is now made in the ascending order.

\section{B. Simulated Annealing}

Simulated Annealing (SA) is an optimization metaheuristic inspired from the annealing process in metallurgy and is a variant of the neighborhood search technique. This is our first attempt to solve the problem of optimizing the experimental throughput; hence we have decided to use SA because of its simplicity, although, in our experience, a Tabu Search meta-heuristic may produce better results. The right choice of optimization approach depends on the particular problem, and we plan to investigate which is the best approach in our future work. SA randomly selects a solution from the neighbors of the current solution. The new solution is then evaluated and accepted if an improvement in cost is achieved. Contrary to the typical neighborhood search technique, SA also accepts deteriorations in cost to a limited extent in an effort to achieve the global optimum [6]. The deterioration in the cost function is accepted with a probability, that depends on the deterioration magnitude and a control parameter called temperature.

An important aspect of SA is the generation of a new solution from the current one. SA uses design transformations ("moves") to transform the current solution $\left(\mathscr{P}^{\text {now }}, S^{\text {now }}\right)$ in

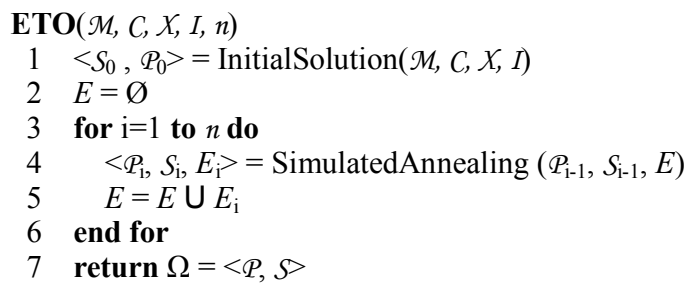

Fig. 3: Optimization Strategy. order to explore the design space. There are three types of moves: (i) moves that change the inputs $S_{R}$ and $S_{C}$, (ii) moves that change $S_{P}$, i.e., how these inputs are fed into the cell culture chamber, and (iii) moves that change placement $\mathcal{P}$ of the colonies on the chamber.

(i) For changing the matrix $S_{C}$ of $I \times M^{\prime}$ elements, we randomly select two elements (compounds $F_{i}$ and $F_{j}$ ) and swap them. These are the type of moves performed most often, since they have a large impact on the generation of new combinations of compounds applied to the cell colonies. Similar moves are performed on $S_{R}$. (ii) Moves that transform $S_{P}$ are applied when the value of the temperature goes below 1 , significantly reducing the acceptance probability of the deteriorated cost solutions. The temperature is thus reset and a random move in $S_{P}$ is made. Since $S_{P}$ has only 0 or 1 as member elements, the maximum number of unique moves is limited. (iii) Moves in $\mathcal{P}$ are applied the least often, only when moves in $S_{P}$ have been exhausted. A move in the $N^{\prime} \times M^{\prime}$ matrix $\mathscr{P}$ is performed by randomly selecting the elements $\left(C_{i} \neq C_{j}\right)$ and swapping them with each other.

The algorithm stops if either the maximum number of unique experiments obtained in one stage considering the biochip capacity is found or the termination time is reached.

\section{EXPERIMENTAL EVALUATION}

In order to evaluate our Experimental Throughput Optimization (ETO) approach, we have performed two sets of experiments. The algorithms were implemented in $\mathrm{C}++$, running on Lenovo T400s ThinkPad with Core 2 Duo Processors at $2.53 \mathrm{GHz}$ and $4 \mathrm{~GB}$ of RAM.

For comparison purposes during evaluation, we have also implemented a straightforward (SF) approach. This is an approach that a good engineer would use when no optimization tools are available. SF starts from an initial solution obtained with the Descend-Ascend scheme given in Section IV.A and then performs a left-to-right roll on the placement $\mathcal{P}$ and the contents of the schedule $S$ in the hope to increase the number of unique combinations, e.g., in Fig. $2 b$ we obtain the stage design $\Omega_{2}$ by rolling over the settings in Fig. 2a. Next, a top-to-bottom roll is performed. Since the top-to-bottom roll cannot be performed on $S_{P}$, it is rolled to the right instead, as shown in the transition from the settings in Fig. $2 b$ to Fig. 2c. For each experimental stage, SF alternately performs a left-to-right and top-to-bottom roll, until termination criteria is reached. On termination, SF returns the best solution found, i.e., the one that maximized the system throughput. In the first set of experiments shown in Table I, we are interested in evaluating the quality of ETO in terms of its ability to maximize the experimental throughput across all desired stages. We have used a total of 9 experimental settings as presented in column 1 to 4 in Table I, ranging the experimental chip area from $6 \times 6$ to $14 \times 14$, the number of compounds from 2 to 8 , the number of cell colonies from 2 to 9 and the number of experimental stages from 2 to 11 . The number of compounds per exposure sequence $I$ was set to 3 for all cases. We have determined the combinations of parameters (each row in the table) such that all possible combinations of compounds for each cell colony may be achievable within the imposed chip area and the 
TABLE I: Full FACTORIAL DESIGN

\begin{tabular}{|c|c|c|c|c|c|c|c|}
\hline \multirow{5}{*}{$N^{\prime} \times M^{\prime}$} & & & & SF & $\begin{array}{c}\text { Best } \\
\text { ETO }\end{array}$ & $\begin{array}{c}\text { Average } \\
\text { ETO }\end{array}$ & \\
\hline \multirow{3}{*}{$6 \times 6$} & $|X|$ & $|C|$ & $n$ & $\begin{array}{c}\gamma \\
(\%)\end{array}$ & $\begin{array}{c}\gamma \\
(\%)\end{array}$ & $\begin{array}{c}\gamma \\
(\%)\end{array}$ & $\begin{array}{c}\text { Standard } \\
\text { Deviation }\end{array}$ \\
\cline { 3 - 8 } & 2 & 9 & 2 & 50 & 83.3 & 78.7 & 3.46 \\
\hline & 3 & 7 & 6 & 43.9 & 88.4 & 84.2 & 3.04 \\
\hline \multirow{3}{*}{$10 \times 10$} & 4 & 5 & 9 & 27.8 & 82.8 & 77.8 & 3.43 \\
\hline & 4 & 3 & 2 & 12.5 & 78.1 & 74.3 & 2.89 \\
\hline & 5 & 2 & 3 & 42.4 & 85.2 & 78.4 & 2.57 \\
\hline \multirow{3}{*}{$14 \times 14$} & 6 & 3 & 7 & 48.3 & 74.8 & 71.8 & 2.17 \\
\cline { 2 - 8 } & 6 & 3 & 4 & 44.9 & 76.2 & 73.5 & 1.72 \\
\cline { 2 - 8 } & 7 & 4 & 7 & 22.4 & 68.9 & 66.8 & 1.36 \\
\hline
\end{tabular}

TABLE II: FRACTIONALly FACTORIAL DESIGN

\begin{tabular}{|c|c|c|c|c|c|}
\hline \multirow{2}{*}{$|X|$} & \multirow{6}{*}{$|C|$} & $|\mathrm{E}|$ & $\gamma(\%)$ & $|\mathrm{E}|$ & $\gamma(\%)$ \\
\cline { 3 - 6 } & \multicolumn{5}{|c|}{$N^{\prime} \times M^{\prime}=6 \times 6, n=3, I=5$} \\
\hline 2 & 5 & 30 & 27.7 & 89 & 82.4 \\
\hline 2 & 6 & 36 & 33.3 & 97 & 89.8 \\
\hline 2 & 7 & 36 & 33.3 & 102 & 94.4 \\
\hline 2 & 8 & 36 & 33.3 & 104 & 96.2 \\
\hline 2 & 9 & 54 & 50 & 106 & 98.1 \\
\hline 2 & 10 & 60 & 55.5 & 106 & 98.1 \\
\hline 3 & 2 & 36 & 33.3 & 108 & 100 \\
\hline 3 & 3 & 36 & 33.3 & 108 & 100 \\
\hline
\end{tabular}

number of stages. It is important to note here that even if the chip capacity is equal to (or more than) the maximum possible combinations of compounds for all cell colonies placed on the chip, $100 \%$ throughput might still not be achievable. This is because the compounds that we feed into the chip affect an entire row (or column), thus there might be situations where a schedule and placement that would guarantee $100 \%$ throughput across all stages does not exist.

Table 1 gives the throughput $\gamma$ achieved by SF and ETO. ETO generated solutions provide experimental throughput as high as $88 \%$ and it does not go below $65 \%$ in any of the cases. As shown, ETO performs significantly better than SF. Together with the best solution, Table I also presents the average and the standard deviation obtained after 10 runs of ETO, exploring the solution space differently in every run. As presented, the standard deviation is quite small indicating that ETO consistently finds solutions that are close to the best solution. We have used a time limit of 10 minutes for ETO.

We have also evaluated our proposed approach for the case when the maximum possible number of combinations exceeds the capacity of the available number of chips. A real world example would be that of the fractionally factorial experimental design performed in the first phase of drug discovery, as discussed in Section I. Table II presents the results. We have considered an active biochip area of $6 \times 6$ with $n=3$ stages and have progressively increased the number of combinations by varying the number of compounds $|X|$ and the number of cell colonies $|C|$. I was set to 5 for all cases. As we can see from Table II, ETO can get close to the $100 \%$ throughput in most of the cases, which means that the biochip is utilized to its full capacity over all the $\mathrm{n}$ stages.

In order to determine the quality of our SA-based ETO strategy, we have used an exhaustive search to determine the optimal solutions. Since the runtime of the exhaustive search is prohibitively large, we were only able to run it for smaller examples, lines 1, 4 and 7 in Table I. In these cases, our ETO approach is capable of obtaining solutions which are very close to the optimum. For the experimental setups in lines 1, 4 and 7 , the difference in terms of the cost function is only $1.4 \%, 2.1 \%$ and $2.3 \%$, respectively.

\section{CONCLUSIONS}

In this paper we have presented a Simulated Annealing based approach in order to design high throughput experiments for cell culture microfluidic biochips. The proposed approach considers multiple parameters (e.g., chip size, number of cell colonies, and number of soluble compounds) as inputs and generates design settings (placements and schedules) for the desired number of experimental stages such that the system throughput is maximized. Multiple experimental setups have been used for evaluating the effectiveness of the proposed approach. We have shown that by optimizing the experimental design, significant improvements in the experimental throughput (and chip utilization) can be achieved, thus increasing the system productivity, saving time and reducing costs.

\section{REFERENCES}

[1] J. El-Ali, P.K. Sorger, and K.F. Jensen, "Cells on Chips," Nature, 442, 403 - 411, 2006.

[2] M.W. Lutz et al., "Experimental design for high-throughput screening," Drug Discovery Today, 1(7), 277-286, 1996.

[3] V. Ts. Vanchikov, "Special form of laminar liquid flow in hydraulic devices," Russian Engineering Research, 28(9), 854-855, 2008.

[4] P. Skafte-Pedersen et al., "A user-friendly, self-contained, programmable microfluidic cell culture system for high quality microscopy," MicroTAS, 2010.

[5] A. Stacey and G. Stacey, Routine quality control testing of cell cultures - Antiviral Methods and Protocols (Part 3), Springer 2000.

[6] E. Aarts, J. Korst, and W. Michiels, Simulated Annealing Search Methodologies, (Part 7), Springer 2005.

[7] T. Thorsen, S. Maerki, and S. Quake, "Microfluidic large-scale integration," Sci, 298, 580-584, 2002.

[8] T. Zhang, K. Chakrabarty, and R. B. Fair, Microelectrofluidic Systems: Modeling and Simulation, CRC Press, 2002.

[9] E.S.K. Cho, H. Moon, and C.J. Kim, "Creating, transporting, cutting, and merging liquid droplets by electrowetting-based actuation for digital microfluidic circuits," Journal of Microelectromechanical Systems, 12(1), 2003.

[10] N. Amin, W. Thies, and S. Amarasinghe, "Computer-aided design for microfluidic chips based on multilayer soft lithography," Proc. of the Intl. Conference on Computer Design, 2-9, 2009.

[11] P.S. Dittrich and A. Manz, "Lab-on-a-chip: microfluidics in drug discovery," Nature Reviews, Drug Discovery 5, 210-218, 2006

[12] G. Sittampalam, S. Kahl, and W. Janzen, "High-throughput screening: advances in assay technologies," Curr. Opin, Chem. Bio, 1, 384, 1997.

[13] R.J. Taylor et al., "Dynamic analysis of MAPK signaling using a high-throughput microfluidic single-cell imaging platform," Proc. of the Natl. Acad. of Sciences, 106, 3758-3763, 2009,

[14] Z. Wang, M. Kim, M. Marquez, and T. Thorsen, "High-density microfluidic arrays for cell cytotoxicity analysis," Lab Chip, 7, 740-745, 2007.

[15] M.E. Kempner, and R.A. Felder, "A review of cell culture automation," The Journal of the Association for Laboratory Automation (JALA), 7(2), 56-62, 2002. 